\title{
ДЕТЕРМИНАНТЫ СОЗДАНИЯ АКЦИОНЕРНОЙ \\ СТОИМОСТИ РОССИЙСКИМИ КОМПАНИЯМИ
}

\author{
Анкудинов А.Б. ${ }^{1}$, Лебедев О.В. ${ }^{2}$
}

В работе представлены результаты эмпирического исследования детерминантов создания акционерной стоимости. Анализ основан на панельных данных; выборка сформирована по данным 107 крупнейших российских публичных компаний нефинансового сектора за период 2004-2012 годов. Метод исследования состоял из двух частей: сначала был проведен регрессионный анализ детерминантов создания акционерной стоимости в терминах стоимостных мультипликаторов цена/балансовая стоимость, цена/прибыль, а также совокупной акционерной доходности, затем проведена оценка детерминантов вероятности превышения стоимостными мультипликаторами среднерыночных показателей и получения совокупной акционерной доходности выше средней по рынку. Результаты исследования показывают, что макроэкономическая конъюнктура выступает более мощным детерминантом создания акционерной стоимости по сравнению с индивидуальными характеристиками компании. Доходность активов, леверидж, инвестиционные расходы, уровень рисковости компании позитивно коррелированы с индикаторами создания акционерной стоимости. Государственные компании торгуются с некоторым дисконтом, а представленные в структуре собственности крупным акционером-нерезидентом - с некоторой премией к рынку. Размер, возраст компании, отраслевые эффекты статистически значимо связаны с индикаторами создания акционерной стоимости. Индивидуальные характеристики компаний имеют большое значение в относительной оценке стоимости компании рынком и существенно меньшее значение в объяснении совокупной акционерной доходности.

Ключевые слова: акционерная стоимость, акционерная доходность, стоимость компании, драйверы стоимости

JEL: G30, G32

\section{Введение}

В настоящее время концепция ориентированного на стоимость управления является доминирующей управленческой парадигмой, в соответствии с которой деятельность компании должна проецироваться на обеспечение устойчивого роста акционерной стоимости в долгосрочной перспективе, а процесс принятия управленческих решений - основываться на драйверах создания стоимости. При этом, если теоретико-концептуальные исследования проблемы создания акционерной стоимости представлены в научной литературе в изобилии, то эмпирическит работы, особенно по данным российских компаний, достаточно редки. В данном контексте целью нашего исследования выступила относительная оценка рычагов создания акционерной стоимости на российском рынке капитала. Прикладная значимость подобного анализа заключается в том, что он помогает менеджерам высветить драйверы стоимости компании на несовершенном финансовом рынке.

В данной работе представлен эконометрический анализ детерминант создания акционерной стоимости. Процесс создания акционерной стоимости изучается в терминах стоимостных мультипликаторов цена/балансовая стоимость (Market to Book Ratio), цена/прибыль (PriceEarnings Ratio), а также совокупной акционерной доходности (Total Shareholder Return). Показатель совокупной акционерной доходности (далее по тексту - «акционерная доходность») подробно анализируется в основополагающих трудах по стоимостному управлению (Rappaport, 1998). Что касается мультипликаторов, то в литературе по стоимостной оценке устоялась формулировка, утверждающая, что рыночная стоимость компании отличается от et балансовой стоимости на величину чистой приведенной стоимости будущих сверхприбылей, то есть скорректированных на риск прибылей выше среднерыночных. Из этого следует, что

1. Ассистент, кафедра корпоративных финансов, Казанский федеральный университет.

2. Авторы готовы по запросу предоставить сформированную панель данных. 
стоимостные мультипликаторы будут отражать оценку инвесторами будущих сверхдоходов, другими словами, спред между ожидаемой доходностью и стоимостью капитала. Использование стоимостных мультипликаторов весьма распространено в эмпирических работах по анализу процесса создания акционерной стоимости (Pandey, 2005; Malighetti et al., 2011).

Изучаемые нами детерминанты создания акционерной стоимости в соответствии с предыдущими исследованиями по оценке стоимости компаний (Bartov et al., 2002; Gou et al., 2005) и доступностью исходных данных были разделены на три группы: финансовые драйверы, структура собственности, прочие контрольные переменные (см. рисунок 1).

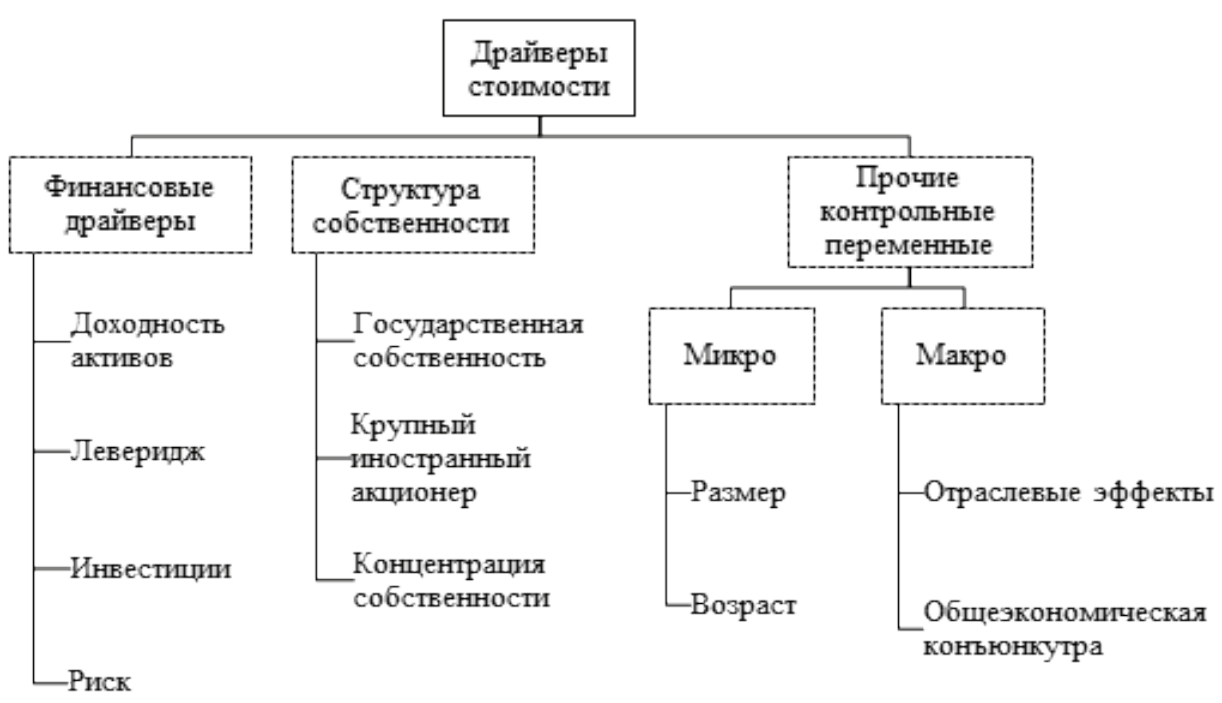

Рисунок 1. Исследуемье драйверы создания акиионерной стоимости

\section{Обзор литературы}

Отметим результаты предыдущих эмпирических исследований детерминантов создания акционерной стоимости в рамках каждой из выделенных групп драйверов стоимости. Наиболее широко в научной литературе представлены результаты эконометрического моделирования влияния финансовых драйверов на индикаторы создания акционерной стоимости. Так, А. Клейдон утверждает, что высокая доля изменений рыночных цен на акции может быть объяснена изменениями величины ожидаемых денежных потоков, и что наиболее репрезентативными переменными динамики рыночной стоимости компании являются доходность активов и инвестиции (Kleidon, 1986). Б. Холл показывает значимую позитивную связь рыночной стоимости компании от отношения денежного потока к капиталу, отношения расходов на НИОКР к капиталу, темпов роста продаж, отношения расходов на рекламу к капиталу (Hall, 1993). И. Панди находит сильную позитивную зависимость мультипликатора цена/балансовая стоимость и экономической рентабельности, делового и финансового рисков (Pandey, 2005). П. Чен и Г. Жэнг показывают, что акционерная доходность наиболее тесно связана с уровнем доходности активов, инвестиционной активностью, изменениями в прибыльности, возможностями роста, изменениями в стоимости капитала (Chen and Zhang, 2007).

Из немногих известных авторам данного исследования эмпирических работ по анализу финансовых драйверов создания акционерной стоимости, выполненных по данным российских компаний, отметим две работы. И.В. Ивашковская и Е.Б. Кукина в своем исследовании используют в качестве индикатора создания акционерной стоимости не рыночные индикаторы, а показатель экономической добавленной стоимости и называют следующие его детерминанты: капитальные вложения, темп роста доходов компании, операционная рентабельность, выплаченные дивиденды (Ивашковская? Кукина, 2009). А.Б. Анкудинов и Л.А. Сафина показывают, что инвестиции в инновации имеют «локомотивный» эффект и позитивно влияют на рыночную стоимость компании (Ankudinov and Safina, 2011). 
Также в литературе представлены эмпирические исследования, направленные на изучения зависимости структуры собственности и стоимости компании. Р. Ла Порта и др. демонстрируют, что стоимость компаний выше в странах с более эффективной защитой миноритарных акционеров и позитивно связана с уровнем концентрации собственности (La Porta et al., 2002). Т. Педерсен и С. Томсен показывают, что статус принадлежности государству может ассоциироваться с более низкой относительной рыночной оценкой собственного капитала компании и что уровень концентрации собственности негативно влияет на стоимость государственных компаний, но концентрация собственности не влияет на стоимость компаний, которые находятся во владении частных лиц (Pedersen and Thomsen, 2003). Также имеются эмпирические свидетельства о потенциальном негативном влиянии конфликта интересов стейкхолдеров «государство» и «акционер» в государственных компаниях на их капитализацию (Chua et al., 2007; Meoli et al. 2009).

Кроме того, значительное влияние на процесс создания акционерной стоимости могут оказывать прочие факторы микро- и макроуровня. Эмпирические работы показывают, что специфичные для отраслей операционные факторы, размер и возраст компании выступают важными детерминантами создания акционерной стоимости (Fama and French, 1996; Chua et al., 2007; Malighetti et al., 2011). Процесс создания акционерной стоимости также подвержен воздействию страновых специфических характеристик: так, Ю. Баи и К. Грин демонстрируют, что экономическая конъюнктура, развитие финансового сектора, правовая среда статистически значимо влияют на акционерную доходность (Bai and Green, 2011).

\section{Гипотезы и выбор прокси-переменных}

На основе обобщения результатов предыдущих эмпирических исследований были сформулированы рабочие гипотезы. В рамках группы финансовых драйверов тестируется связь с созданием акционерной стоимости доходности активов, левериджа, инвестиционной активности, уровня рисковости компании. Основным источником создания стоимости на конкурентных продуктовых рынках выступает наличие устойчивых конкурентных преимуществ, позволяющих эксплуатировать экономические ренты. Доходность активов может выступать косвенным индикатором наличия конкурентных преимуществ компании (Olsen et al., 2006). Предыдущие эмпирические исследования по развитым рынкам капитала обнаруживают позитивную зависимость между доходностью активов и способностью создания акционерной стоимости (Kleidon, 1986; Chen and Zhang, 2007). Логичным представляется также предположение о высоком уровне информационного контента показателей учетной доходности активов в условиях характеризующегося достаточно короткими временными горизонтами отечественного финансового рынка. Таким образом, формально гипотеза 1 была сформулирована следующим образом:

H1: Уровень доходности активов оказывает положительное влияние на процесс создания акционерной стоимости.

Политика в области структуры капитала многих финансово благополучных отечественных компаний консервативна и большая опора на долговое финансирование во многих случаях способна снизить стоимость капитала компании. Кроме того, финансовый леверидж может выступать инструментом дисциплинирования менеджмента и снижать агентские затраты. Имеются эмпирические свидетельства подобной зависимости между финансовой политикой и созданием акционерной стоимости (Margaritis and Psillaki, 2010). Вместе с тем более высокая рыночная стоимость компании часто ведет к более низким издержкам по обслуживанию заемного капитала, что обусловливает более агрессивную финансовую политику и позитивную корреляцию между стоимостными мультипликаторами и уровнем левериджа (Chen and Zhao, 2006). Таким образом, в рамках гипотезы 2 предполагается:

H2. Уровень финансового левериджа положительно влияет на создание акционерной стоимости.

Создание акционерной стоимости - это продукт в первую очередь состоятельных инвестиционных решений, генерирующих экономические ренты. В научной литературе имеются эм- 
пирические свидетельства прямой зависимости инвестиционных расходов и создания акционерной стоимости (Hall, 1993; Chen and Zhang, 2007; Ивашковская и Кукина, 2009). На основе предположения о том, что менеджеры в большинстве случаев движимы именно стремлением к максимизации акционерной стоимости, гипотеза 3 была сформулирована следующим образом:

Н3. Уровень инвестиционной активности позитивно влияет на создание акционерной стоимости.

Связь исследуемых индикаторов создания акционерной стоимости и уровня рисковости компании не столь тривиальна. С одной стороны, более высокий риск ведет к более высокой стоимости капитала и, следовательно, к меньшей рыночной стоимости (отрицательная корреляция). С другой стороны, более высокий риск означает и более высокую доходность в будущем, которая не контролируется переменной доходности активов за текущий период (позитивная корреляция) (Koller et al., 2010). Мы предполагаем, что последний фактор будет оказывать большее влияние. За неимением данных для расчета более информативных индикаторов риска, в качестве прокси уровня риска в нашем исследовании выступает коэффициент вариации выручки компании за весь период исследования. Гипотеза 4 определяется следующим образом:

H4: Уровень риска оказывает положительное влияние на создание акционерной стоимости.

Вторая группа исследуемых детерминантов создания акционерной стоимости описывает структуру собственности. В нее были включены переменные принадлежности государству, наличия крупного иностранного акционера, концентрации собственности. Зависимость между присутствием в структуре собственности государства и созданием акционерной стоимости неоднозначна. С одной стороны, государственные компании могут лоббировать выгодные для них законодательные инициативы, иметь преимущества в получении различных лицензий. С другой стороны, инвесторы отдают себе отчет в том, что в государственных компаниях интересы стейкхолдера «государство» зачастую превалируют над интересами стейкхолдера «акционер» и максимизация акционерной доходности может не являться для таких компаний приоритетной. Представленные в литературе результаты исследований демонстрируют негативную зависимость фактора государственной собственности и капитализации компаний (Pedersen and Thomsen, 2003; Meoli et al., 2009; [Malighetti et al., 2011). Таким образом, гипотеза 5 предполагает:

H5: Статус принадлежности государству негативно влияет на процесс создания акционерной стоимости.

Наличие иностранного акционера в структуре собственности может выступать косвенным индикатором потенциального внедрения системы управления на основе критерия стоимости и более широкого использования финансовых инструментов в целях дисциплинирования менеджмента. Ряд эмпирических работ демонстрируют, что сам факт внедрения системы стоимостного управления ассоциируется с получением акционерной доходности выше среднерыночной (Hogan and Lewis, 2005; Rapp et al., 2011). В нашем исследовании гипотеза 6 была сформулирована следующим образом:

H6: Наличие иностранного акционера в структуре собственности положительно влияет на процесс создания акционерной стоимости.

Что касается влияния концентрации собственности на создание акционерной стоимости, то для развитых рынков с преимущественно распыленной структурой собственности исследования показывают позитивное влияние уровня концентрации собственности на рыночную стоимость компании (La Porta et al., 2002; Pedersen and Thomsen, 2003). Авторы объясняют подобную зависимость тем, что несколько акционеров могут консолидировать контроль и сконцентрироваться на максимизации акционерной доходности. В то же время для отечественного рынка с концентрированной структурой собственности проблема консолидации контроля не столь актуальна, и чрезмерно высокая концентрация может уже обусловливать 
пренебрежение интересами миноритарных акционеров. Таким образом, гипотеза 7 определяется следующим образом:

H7: Уровень концентраџии собственности негативно воздействует на создание акционерной стоимости.

В качестве контрольных переменных выступали размер, возраст компании, отраслевые эффекты и общеэкономическая конъюнктура. Акции крупных компаний, как правило, более ликвидны и могут относиться к «голубым фишкам», за что инвесторы часто готовы платить премию. Соответственно, гипотеза 8 предполагает:

Н8: Размер компании оказывает положительное влияние на создание акционерной стоимости.

Возраст компании как индикатор стадии жизненного цикла компании, при прочих равных, может сигнализировать о наличии привлекательных инвестиционных возможностей. Имеется ряд исследований, направленных на изучение связи роста фирмы и возраста (Farinas and Moreno, 2000; Huergo and Jaumandreu, 2004), также по российским компаниям (Сафиуллин и др., 2013). В частности, в представленных в литературе результатах эмпирических исследований обнаружена прямая зависимость размера и обратная зависимость возраста и рыночной стоимости компании (Malighetti et al., 2011). Таким образом, гипотеза 9 была сформулирована следующим образом:

\section{H9: Возраст компании отрицательно влияет на создание акциионерной стоимости.}

Важную роль в создании акционерной стоимости могут играть отраслевые эффекты и общеэкономические факторы (Olsen et al., 2006). В рамках нашего исследования для учета отраслевых эффектов и макроэкономической конъюнктуры в исследуемые модели были включены соответствующие группы дамми-переменных отраслевой принадлежности и времени (года).

\section{База данных и описание переменных}

Выборка была сформирована на основе данных по крупнейшим по выручке российским публичным компаниям нефинансового сектора за девятилетний период - 2004-2012 годы; в выборку были включены данные по 107 компаниям, имеющим листинг на ММВБ. Подобный подход к формированию панели данных на основе крупных компаний обусловлен тем, что акции небольших компаний менее ликвидны и их рыночные котировки часто не репрезентируют процесс создания акционерной стоимости. Анализ же именно панельных данных обусловлен как необходимостью принятия в учет ненаблюдаемой разнородности между компаниями (unobserved heterogeneity), так и необходимостью расширения выборки.

Источником используемых в настоящем исследовании данных выступили «Система профессионального анализа рынков и компаний СПАРК» ${ }^{1}$ в части данных финансовой отчетности (в соответствии с российскими стандартами бухгалтерского учета), регистрационных данных, а также информационный портал ММВБ в части рыночных данных по стоимости акций компаний. Анализ основывался на годовых данных, однако по некоторым переменным данные за отдельные годы отсутствовали: ряд компаний стали публичными позднее 2004 года или же, напротив, прекратили листинг; отрицательные и абнормально высокие значения по стоимостным мультипликаторам исключались из выборки. Поскольку количество наблюдений для каждой компании различно, панельные данные являются несбалансированными (unbalanced panel)2. Однако отметим, что оценки состоятельны, если характер отсутствующих данных случаен, то есть вероятность отсутствия не зависит от величины переменной, данные по которой отсутствуют.

Описательная статистика и порядок расчета используемых в моделировании переменных представлены в таблице 1. Дамми-переменные государственной собственности и наличия крупного иностранного акционера были сформированы на основе Общероссийского клас-

1. Система основывается на данных, раскрываемых компаниями, данных федеральных ведомств (Росстат, ФНС), данных СМИ (подробнее см. www.spark-interfax.ru).

2. Авторы готовы по запросу предоставить сформированную панель данных. 
сификатора форм собственности ОКФС. Возраст компании рассчитывался исходя из даты государственной регистрации компании как юридического лица (мы отдаем себе отчет в возможных ограничениях подобного анализа, поскольку рыночный период в России очерчен лишь чуть более чем 20 годами, и многие компании не настолько молоды, как указывают их регистрационные данные). Отраслевая структура анализируемых компаний на основе укрупненных разделов Общероссийского классификатора видов экономической деятельности (ОКВЭД) следующая: добыча полезных ископаемых - 19\%, обрабатывающие производства $-25 \%$, производство и распределение электроэнергии, газа и воды $-42 \%$, торговля и услуги $-5 \%$, транспорт и связь $-9 \%$.

Для всего периода средние значения мультипликаторов цена/балансовая стоимость и цена/ прибыль составили 1,21 и 11,6 соответственно, акционерной доходности - 13\%. Переменные достаточно волатильны (о чем можно судить по высокому значению коэффициента вариации, то есть отношения стандартного отклонения к среднему значению). Высокие докризисные значения существенно снизились во время кризиса, и затем последовала некоторая коррекция (см. рисунок 2).

Таблица 1

\begin{tabular}{|c|c|c|c|c|c|}
\hline \multicolumn{6}{|c|}{ Описательная статистика, 2004-2012 годы } \\
\hline Переменная & Описание & Среднее & СКО & Min & Max \\
\hline $\begin{array}{l}\text { Цена / балансо- } \\
\text { вая стоимость }\end{array}$ & $\begin{array}{c}\text { Капитализация / бухгалтерская стои- } \\
\text { мость собственного капитала }\end{array}$ & 1,21 & 0,65 & 0,28 & 2,63 \\
\hline Цена / прибыль & Капитализация / чистая прибыль компании & 11,60 & 7,83 & 1,76 & 32,69 \\
\hline $\begin{array}{c}\text { Акционерная до- } \\
\text { ходность }\end{array}$ & Темп прироста капитализации + дивидендная доходность & 0,13 & 0,58 & $-0,92$ & 1,92 \\
\hline Доходность активов & Чистая прибыль / совокупные активы & 0,07 & 0,09 & $-0,25$ & 0,37 \\
\hline Инвестиции & Темп прироста стоимости основных средств & 0,19 & 0,31 & $-0,36$ & 1,73 \\
\hline Леверидж & Доля долга в структуре финансирования & 0,46 & 0,25 & 0,00 & 0,92 \\
\hline Риск & Коэффициент вариации выручки за 2004-2012 гг. & 0,52 & 0,24 & 0,13 & 1,27 \\
\hline Гос. собственность & $\begin{array}{l}\text { Дамми-переменная, }=1 \text { если акционером } \\
\text { является государство; =0 в ином случае; }\end{array}$ & 0,37 & 0,48 & 0,00 & 1,00 \\
\hline Иностранный акционер & Наличие крупного иностранного акционера (1=да; $0=$ =ет) & 0,36 & 0,48 & 0,00 & 1,00 \\
\hline $\begin{array}{l}\text { Концентрация } \\
\text { собственности }\end{array}$ & Доля владения трех крупнейших акционеров & 0,81 & 0,17 & 0,32 & 0,99 \\
\hline Размер & Натуральный логарифм выручки компании & 23,77 & 1,94 & 13,69 & 28,93 \\
\hline Возраст & Количество лет со дня регистрации компании & 10,32 & 5,83 & 1,00 & 22,00 \\
\hline
\end{tabular}

Доходность активов в среднем равна приблизительно 7\%, темп прироста стоимости основных средств - 19\%, доля заемных средств в структуре источников финансирования - $46 \%$. В структуре собственности $37 \%$ и $36 \%$ компаний соответственно государство и нерезиденты представлены в качестве крупных акционеров. В среднем доля владения трех крупнейших акционеров равна 81\%. Средний возраст компаний составил около 10 лет. Хотя ряд независимых переменных также существенно волатильны (доходность активов, инвестиции), в целом рыночные показатели, выступающие в качестве зависимых переменных, характеризуются большей волатильностью. 

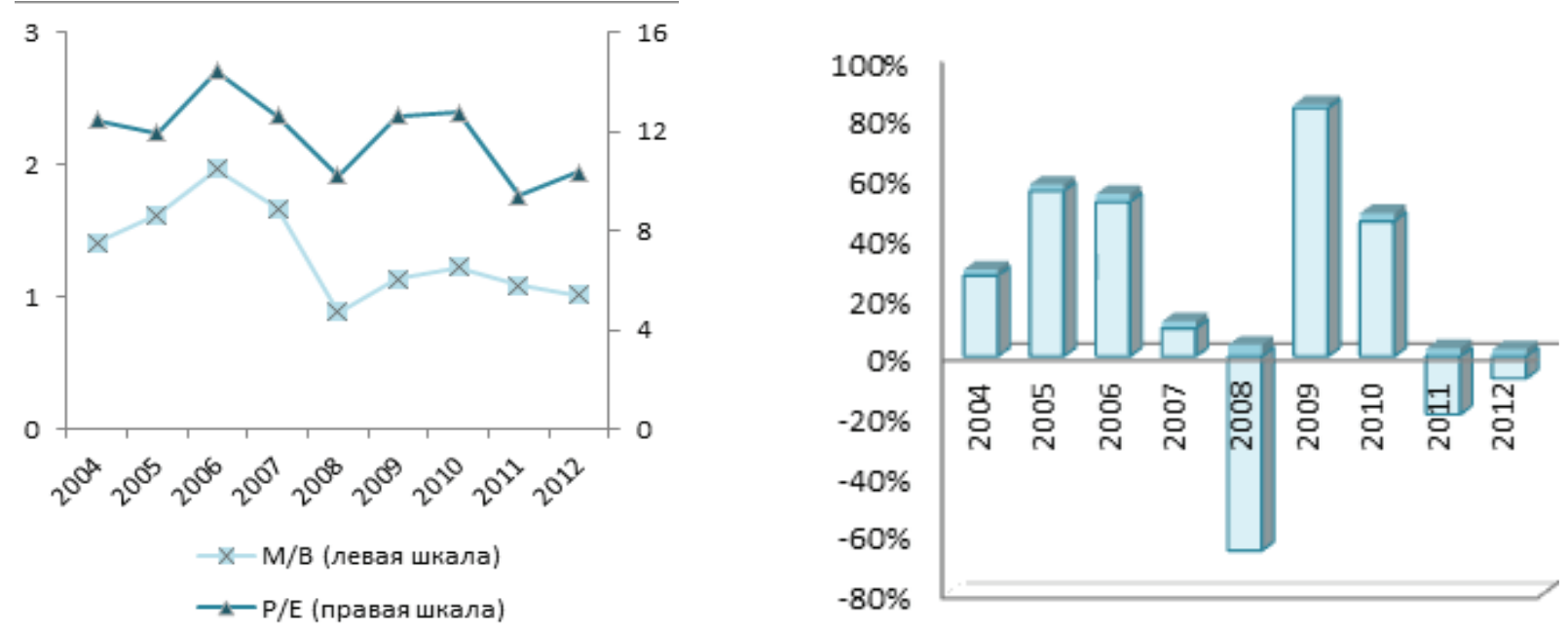

Рисунок 2. Динамика средних значений стоимостных мультипликаторов и акционерной доходности

\section{Метод исследования}

Проведенный в рамках настоящего исследования анализ состоит из двух частей. В первой части проанализированы детерминанты создания акционерной стоимости в терминах показателя акционерной доходности и стоимостных мультипликаторов цена/балансовая стоимость и цена/прибыль. Однако, как будет показано ниже, создание стоимости существенно детерминируется макроэкономическими факторами, поэтому во второй части проведена оценка детерминант вероятности превышения стоимостными мультипликаторами среднерыночных показателей и получения акционерной доходности выше средней по рынку. Для этого исследуемые зависимые переменные были модифицированы в бинарные переменные (если значение зависимой переменной за определенный период превышало среднее значение за этот период, то бинарная переменная приравнивалась единице, в ином случае - нулю). Затем было проведено Probit-моделирование детерминант превышения стоимостными мультипликаторами среднерыночных показателей и получения сверхдоходности, то есть доходности выше среднерыночной (однако на риск сама зависимая переменная не корректировалась; учет риска проводился через включения в модели соответствующей независимой переменной).

В рамках регрессионного анализа детерминанты создания акционерной стоимости исследовались с использованием трех классов моделей (Cameron and Trivedi, 2009):

1. Модели со случайными эффектами (RE)

$y_{i t}=X_{i}^{\prime} \beta+\alpha_{i}+\varepsilon_{i}$,

где $\mathrm{X}$ - вектор независимых переменных, $\beta$ - оцениваемые параметры. Случайные эффекты $\alpha_{i}$ и ошибки $\varepsilon_{i t}$ здесь - независимые одинаково распределенные случайные величины $\alpha_{i} \sim \operatorname{iid}\left(0, \sigma_{\alpha}^{2}\right)$ и $\varepsilon_{i} \sim \operatorname{iid}\left(0, \sigma_{\varepsilon}^{2}\right)$, которые независимы от $x_{i 1}, \ldots x_{t}$. Использование RE-моделей позволяет получить несмещенные оценки, если ненаблюдаемая разнородность не коррелированна с регрессорами.

2. Модели с фиксированными эффектами (FE):

$y_{i t}-\bar{y}_{i}=\left(X_{i}-\bar{X}_{i}\right)^{\prime} \beta+\varepsilon_{i}-\bar{\varepsilon}_{i}$,

FE-модели позволяют устранить из моделей инвариантные во времени ненаблюдаемые индивидуальные эффекты и решить проблему эндогенности по причине невключения в модели релевантных предикторов. Из этого, однако, следует, что они не позволяют оценить незначительно изменяемые во времени анализируемые нами детерминанты стоимости (такие, как дамми-переменные государственной собственности, присутствия крупного иностранного акционера; отраслевые эффекты). 
3. Сквозные модели (РА). Предполагается, что регрессоры экзогенны, таким образом, производится сквозная FGLS (Feasible Generalized Least Squares) оценка без осуществления декомпозиции общей ошибки $u_{i t}=\alpha_{i}+\varepsilon_{i}$.

Все модели предполагают принятие в учет кросс-корреляций между ошибками для отдельной компании. Попарное сравнение моделей проводилось с использованием: теста Вальда (Wald test) для сравнения моделей с фиксированными эффектами и сквозных регрессий, теста Бройша-Пагана (Breusch-Pagan test) для сравнения моделей со случайными эффектами и сквозных регрессий, теста Хаусмана (Hausman test) для сравнения моделей с фиксированными эффектами и моделей со случайными эффектами.

При оценке же Probit-моделей наблюдаемые зависимые переменные $y_{i t}^{*}$ (совокупная акционерная доходность, стоимостные мультипликаторы цена/балансовая стоимость и цена/прибыль) были трансформированы в бинарные:

$$
y_{i t}= \begin{cases}1, \text { если } & y_{i t}^{*}>\bar{y}_{t} . \\ 0, \text { если } & y_{i t}^{*} \leq \bar{y}_{t},\end{cases}
$$

Были построены Probit-модели со случайными эффектами и сквозные Probit-модели, структура которых соответствует рассмотренным выше. Тестирование Probit-модели со случайными эффектами (RE) против сквозной Probit-модели (PA) производилось с помощью критерия отношения правдоподобия (Likelihood Ratio test, LR).

$\mathrm{C}$ формулами расчета $\beta$ коэффициентов $\mathrm{PA}, \mathrm{RE}, \mathrm{FE}$ линейных регрессий можно ознакомиться в Stata (Stata, 2009, p. 463-466]; $\beta$ и маржинальных эффектов PA и RE Probit-моделей - там же) Stata, 2009, p. 424-426]. Все расчеты и управление данными были произведены с помощью программного пакета STATA12.0.

\section{Анализ результатов}

В таблице 2 представлены результаты регрессионного анализа детерминантов создания акционерной стоимости. Попарное сравнение моделей показывает более высокую статистическую мощность моделей с фиксированными эффектами в объяснении стоимостных мультипликаторов, но не показателя акционерной доходности. Это может свидетельствовать о том, что ненаблюдаемые индивидуальные эффекты компаний имеют большое значение в относительной оценке капитализации компании рынком и существенно меньшее значение в объяснении акционерной доходности. Тем не менее мы приводим оценки двух классов моделей, поскольку ряд независимых переменных инвариантны во времени и не могут быть оценены в FE-моделях, а формальные тесты RE против FE-моделей проводятся лишь по включаемым в оба класса моделей наборам переменных.

Таблий 2

\section{Оценки регрессий со случайными и фиксированными эффектами}

\begin{tabular}{ccccccc}
\hline & \multicolumn{2}{c}{$\begin{array}{c}\text { Цена / балансовая } \\
\text { стоимость }\end{array}$} & \multicolumn{2}{c}{ Цена / прибыль } & \multicolumn{2}{c}{ Акционерная доходность } \\
Переменные & -2 FE & FE & RE & FE & RE & FE \\
\hline Доходность активов & 1,682 & 0,850 & - & - & 1,135 & 0,616 \\
& $(3,22)^{* * *}$ & $(1,65)^{*}$ & & & $(4,43)^{* * *}$ & $(1,84)^{*}$ \\
Леверидж & 1,065 & 0,860 & 2,378 & 9,773 & 0,234 & 0,138 \\
& $(7,17)^{* * *}$ & $(2,37)^{* *}$ & $(1,13)$ & $(1,94)^{*}$ & $(2,73)^{* * *}$ & $(1,65)^{*}$ \\
Инвестиции & 0,122 & 0,086 & 2,399 & 2,156 & 0,022 & 0,026 \\
& $(1,36)$ & $(0,91)$ & $(2,02)^{* *}$ & $(1,76)^{*}$ & $(0,35)$ & $(0,35)$ \\
Риск & 0,426 & - & 7,148 & - & 0,070 & -
\end{tabular}




$\begin{array}{ccccccc}\text { Гос. собственность } & -0,163 & - & 1,210 & - & 0,035 \\ \text { Иностранный акционер } & (1,71)^{*} & & (0,96) & & (1,07) \\ & 0,172 & - & 1,932 & - & 0,014 & - \\ \text { Концентрация собственности } & (1,78)^{*} & & (1,64)^{*} & & (0,40) \\ & 0,079 & - & 3,733 & - & -0,114 & - \\ \text { Размер } & (0,32) & & (1,10) & & (1,36) & 0,048 \\ \text { Возраст } & 0,095 & -0,150 & 0,493 & -0,584 & 0,010 & (0,92) \\ & (3,14)^{* * *} & (1,13) & (0,99) & (0,24) & (1,69)^{*} & -0,035 \\ \text { Отраслевые дамми } & -0,022 & -0,027 & 0,041 & -0,372 & 0,001 & (2,22)^{* *} \\ \text { Дамми времени } & (1,65)^{*} & (0,79) & (0,31) & (0,95) & (0,37) & - \\ \text { Соns. } & + & - & + & - & + & + \\ & + & + & + & + & + & -0,695 \\ \text { N } & -1,614 & 4,582 & -7,619 & 24,972 & -0,124 & (0,58) \\ \mathrm{R}^{2} \text { (within) } & (2,27)^{* *} & (1,51) & (0,55) & (0,43) & (0,55) & 446 \\ \text { Wald / F } & 401 & 401 & 416 & 416 & 446 & 0,57 \\ \text { Вreusch-Рagan / Наusman } & - & 0,46 & - & 0,14 & - & 39,9 * * * \\ & 390,9 * * * & 23,1 * * * & 77,0 * * * & 6,14 * * * & 504,4 * * * & 11,48\end{array}$

Примечания: представлены оценки детерминантов создания акционерной стоимости моделей со случайными (RE) и фиксированными (FE) эффектами. Порядок расчета исследуемых переменных представлен в таблице 1 . В скобках представлены значения $t$-статистик, скорректированных на гетероскедастичность. В модели включены дамми-переменные отраслевой принадлежности (за исключением моделей с фиксированными эффектами) и дамми-переменные времени (года). Статистика Вальда (Wald) и F-статистика используются для тестирования совместной значимости независимых переменных моделей со случайными и фиксированными эффектами соответственно. Тест Бройша-Пагана (Breusch-Pagan) представлен для сравнения моделей со случайными эффектами против сквозных регрессий, тест Хаусмана (Hausman) - для сравнения моделей с фиксированными эффектами и моделей со случайными эффектами.

$* * * \mathrm{p}<0,01, * * \mathrm{p}<0,05, * \mathrm{p}<0,10$

Значения статистики Вальда и F-статистики позволяют отклонить нулевую гипотезу о совместной незначимости факторов RE- и FE-моделей соответственно с высоким уровнем надежности: величины p-value $<0,000$. Следует отметить, что общая макроэкономическая ситуация (исследуемая через дамми-переменные времени), играет первостепенную роль в объяснении динамики индикаторов создания акционерной стоимости. Так, при исключении дамми-переменных времени значение $\mathrm{R}^{2}$ снижается с $46 \%$ до $15 \%$ в модели описания мультипликатора цена/балансовая стоимость, с 14\% до 5\% в модели описания мультипликатора цена/прибыль, с 57\% до 9\% в модели описания акционерной доходности. Это может быть объяснено высокой волатильностью российского рынка капитала, тогда как волатильность фундаментальных переменных создания стоимости значительно ниже.

Переходя к анализу детерминантов акционерной доходности на микроуровне, отметим, что доходность активов компаний оказывает наиболее мощное воздействие на создание акционерной стоимости. Выражаясь формально, согласно оценкам FE-моделей более высокая (на $10 \%)$ доходность активов, при прочих равных, ассоциировалась с большим на 0,085 мультипликатором цена/балансовая стоимость и с большей на 6\% акционерной доходностью ${ }^{1}$. Оценки коэффициентов RE-моделей несколько выше, что может свидетельствовать о том, что переменная не экзогенна и может быть несколько коррелирована с ненаблюдаемыми индивидуальными эффектами (например, с конкурентоспособностью компании, которая может обусловливать более высокую доходность активов, но не полностью отражать потенциал соз-

1. Следует уточнить, что везде далее в обсуждении результатов, когда мы говорим о процентах, мы подразумеваем изменение в процентных пунктах, а не процентное изменение. Например, если акционерная доходность увеличилась с 15\% до 20\%, то изменение процентных пунктов составляет 5\% (20-15\%), в то время как процентное изменение составляет $33 \%$ [(20-15)/15]. 
дания стоимости через данную переменную). Переменная доходности активов исключена из модели, описывающей мультипликатор цена/прибыль, по причине линейной корреляции данных переменных (показатель прибыли использовался при расчете как зависимой, так и независимой переменной).

Как и предполагалось в рамках гипотезы 2, обнаружена статистически значимая прямая зависимость финансового левериджа и индикаторов создания акционерной стоимости. Так, большая на $10 \%$ доля долга в структуре капитала, при прочих равных, обусловливает более высокое значение мультипликаторов цена/балансовая стоимость и цена/прибыль на 0.086 и на 0,97 соответственно (FE-оценки), более высокую на 2,3\% акционерную доходность (REоценка).

Положительная зависимость инвестиционной активности компании и индикаторов создания акционерной стоимости статистически менее надежна. Коэффициент перед соответствующей переменной значим только в моделях, описывающих мультипликатор цена/прибыль. В соответствии с FE-оценками, на 10\% более высокие темпы прироста стоимости основных средств ассоциируются с более высоким на 0,22 значением мультипликатора цена/прибыль. B модели же описания мультипликатора цена/балансовая стоимость RE-оценка коэффициента регрессии становится значимой лишь на $10 \%$ уровне при использовании одностороннего критерия.

Уровень делового риска в терминах волатильности выручки компании прямо связан со стоимостными мультипликаторами. Так, более высокий на 0,25 коэффициент вариации выручки (одно стандартное отклонение) обусловливал более высокие значения мультипликаторов цена/балансовая стоимость и цена/прибыль приблизительно на 0,11 и 1.79 соответственно. В то же время коэффициент перед переменной незначим в модели, описывающей акционерную доходность. Таким образом, если акции более высокого уровня рисковости в среднем и торгуются с некоторой премией к рынку, это не позволяет реализовать более высокую акционерную доходность.

Что касается переменных структуры собственности, то оценки коэффициентов перед соответствующими переменными менее надежны по сравнению с коэффициентами переменных группы «финансовые драйверы». Коэффициент перед дамми-переменными государственной собственности статистически значим лишь в модели, описывающей мультипликатор цена/ балансовая стоимость и, как и предполагалось, имеет отрицательный знак. При прочих равных величина мультипликатора цена/балансовая стоимость ниже для государственных компаний на 0,163. Наличие же иностранного акционера в структуре собственности, напротив, положительно связано с рыночной стоимостью компании. При этом корреляция данных переменных, характеризующих структуру собственности, с величиной акционерной доходности статистически незначима. Уровень концентрации собственности, согласно полученным оценкам, не связан с относительной рыночной оценкой компании, однако негативно коррелирован с величиной акционерной доходности (коэффициент значим на 10\%-ном уровне при использовании одностороннего критерия). Здесь же следует отметить ряд ограничений подобного анализа. Переменные были сформированы на данных, которые не всегда корректно отражают действительные характеристики структуры собственности той или иной компании. Так, иностранным акционером может выступать отечественная компания, зарегистрированная в иностранной (как правило, оффшорной) юрисдикции. А в отношении информации по крупным акционерам данные могут быть представлены лишь по номинальным держателям, а не конечным владельцам.

Направление влияния прочих контрольных переменных также в целом укладывается в рамки выдвинутых гипотез. Так, согласно RE-оценкам, более крупные компании характеризуются более высокими значениями мультипликатора цена/балансовая стоимость и акционерной доходности: повышение выручки компании с 50 до 100 млрд руб., при прочих равных, ассоциировались с более высокими значениями мультипликатора цена/балансовая стоимость на $0,066\left(\left[\ln \left(100 * 10^{9}\right)-\ln \left(50 * 10^{9}\right)\right] * 0,095 \approx 0,066\right)$ и совокупной акционерной доходности на 
$0,7 \%\left(\left[\ln \left(100 * 10^{9}\right)-\ln \left(50 * 10^{9}\right)\right] * 0,048 \approx 0,007\right)$. В то же время, FЕ-оценки становятся статистически незначимыми, что может свидетельствовать о том, что ненаблюдаемые индивидуальные эффекты, важные с точки зрения создания акционерной стоимости, коррелированы с размером компании.

Возраст компании негативно коррелирован с индикаторами создания акционерной стоимости. Так, на 10 лет более зрелая компания, при прочих равных, обеспечивает меньшую на 3,5\%-ную акционерную доходность (FE-оценка). Однако коэффициенты перед переменной статистически ненадежны в моделях, описывающих стоимостные мультипликаторы. Контрольные переменные макроуровня также выступают важными детерминантами создания акционерной стоимости (проводился тест на значимость групп отраслевых дамми-переменных и дамми-переменных времени, показавший их совместную статистическую значимость).

Таблица 3

\begin{tabular}{|c|c|c|c|}
\hline \multicolumn{4}{|c|}{ Оценки Probit-моделей } \\
\hline & $\begin{array}{c}\text { Цена / балансовая } \\
\text { стоимость }\end{array}$ & Цена / прибыль & $\begin{array}{l}\text { Акционерная } \\
\text { доходность }\end{array}$ \\
\hline & $\mathrm{RE}$ & $\mathrm{RE}$ & PA \\
\hline \multirow[t]{2}{*}{ Доходность активов } & 4,010 & - & 2,147 \\
\hline & $(3,17)^{* * *}$ & & $(6,00)^{* * *}$ \\
\hline \multirow[t]{2}{*}{ Леверидж } & 2,904 & 0,433 & 0,195 \\
\hline & $(4,38)^{* * *}$ & $(1,14)$ & $(1,97)^{* *}$ \\
\hline \multirow{2}{*}{ Инвестиции } & 0,716 & 0,250 & 0,097 \\
\hline & $(1,98)^{* *}$ & $(1,03)$ & $(1,20)$ \\
\hline \multirow[t]{2}{*}{ Риск } & 1,167 & 1,139 & 0,125 \\
\hline & $(1,70)^{*}$ & $(2,37)^{* *}$ & $(1,06)$ \\
\hline \multirow[t]{2}{*}{ Гос. собственность } & $-0,397$ & 0,089 & $-0,002$ \\
\hline & $(1,08)$ & $(0,42)$ & $(0,04)$ \\
\hline \multirow[t]{2}{*}{ Иностранный акционер } & 0,639 & 0,538 & $-0,038$ \\
\hline & $(2,20)^{* *}$ & $(2,41)^{* *}$ & $(0,72)$ \\
\hline \multirow[t]{2}{*}{ Концентрация собственности } & 0,005 & 0,770 & $-0,285$ \\
\hline & $(0,01)$ & $(1,37)$ & $(2,15)^{* *}$ \\
\hline \multirow[t]{2}{*}{ Размер } & 0,295 & 0,160 & 0,052 \\
\hline & $(2,46)^{* *}$ & $(2,28)^{* *}$ & $(1,65)^{*}$ \\
\hline \multirow[t]{2}{*}{ Возраст } & $-0,036$ & 0,007 & $-0,002$ \\
\hline & $(0,77)$ & $(0,29)$ & $(0,18)$ \\
\hline Отраслевые дамми & + & + & + \\
\hline Дамми времени & + & + & + \\
\hline $\mathrm{N}$ & 401 & 416 & 446 \\
\hline Wald & $52,3 * * *$ & $32,8 * *$ & $51,2 * *$ \\
\hline LR test & $45,9 * * *$ & $7,38^{* * *}$ & 0,1 \\
\hline
\end{tabular}

Примечания: представлены маржинальные эффекты Probit-моделей со случайными эффектами (RE) и сквозных Probit-моделей (PA) вероятности превышения индикаторами создания акционерной стоимости среднерыночных показателей. Порядок расчета исследуемых переменных представлен в таблице 1. В скобках представлены значения Z-статистик. В модели включены дамми-переменные отраслевой принадлежности и дамми-переменные времени (года). Статистика Вальда (Wald) используется для тестирования совместной значимости независимых переменных моделей. LR-тест представлен для сравнения моделей со случайными эффектами и сквозных моделей.

$* * * \mathrm{p}<0,01, * * \mathrm{p}<0,05, * \mathrm{p}<0,10$

В таблице 3 представлены результаты Probit-оценивания детерминант превышения индикаторами создания стоимости среднерыночных показателей. Полученные в рамках Probitмоделирования результаты совпадают с результатами регрессионного анализа. Так, на 10\% 
более высокая доходность активов, при прочих равных, обусловливает большую вероятность (на 40\%) превышения мультипликатором цена/балансовая стоимость среднерыночного значения и большую вероятность (на 22\%) получения акционерной доходности выше среднерыночной. Влияние переменных, характеризующих структуру финансирования, инвестиционную активность, уровень рисковости, несколько слабее. Оценки перед переменными структуры собственности, как и в регрессионном анализе, менее надежны, а контрольные переменные микро- и макроуровня выступают значимыми детерминантами создания акционерной стоимости.

Получение в рамках Probit-моделирования результатов, не противоречащих регрессионному анализу, весьма логично; более высокое значение индикатора создания акционерной стоимости и обусловливает вероятность превышения им среднерыночного уровня. В то же время подобный анализ позволяет провести дополнительную проверку выдвинутых гипотез, поскольку в данном случае проводится оценка относительного потенциала создания стоимости в контексте общерыночной конъюнктуры.

В таблице 4 обобщены эмпирические результаты тестирования выдвинутых гипотез.

Эмпирические результаты оценки детерминант создания акционерной стоимости

\begin{tabular}{|c|c|c|c|c|}
\hline \multicolumn{4}{|c|}{ Гипотезы } & \multirow{2}{*}{$\begin{array}{c}\text { Результаты } \\
\text { Не отвергается } \\
\text { (p < }<0,01)\end{array}$} \\
\hline H1 & $\begin{array}{c}\text { Конкурентные преимущества } \\
\text { (доходность активов) }\end{array}$ & + & $\begin{array}{c}\text { Создание акционерной } \\
\text { стоимости }\end{array}$ & \\
\hline $\mathrm{H} 2$ & $\begin{array}{c}\text { Структура капитала } \\
\text { (финансовый леверидж) }\end{array}$ & + & $\begin{array}{c}\text { Создание акционерной } \\
\text { стоимости }\end{array}$ & $\begin{array}{c}\text { Не отвергается } \\
(\mathrm{p}<0,05)\end{array}$ \\
\hline $\mathrm{H} 3$ & Инвестиционная активность & + & $\begin{array}{c}\text { Создание акционерной } \\
\text { стоимости }\end{array}$ & $\begin{array}{c}\text { Не отвергается } \\
(\mathrm{p}<0,1)\end{array}$ \\
\hline $\mathrm{H} 4$ & $\begin{array}{c}\text { Уровень рисковости } \\
\text { (волатильность выручки) }\end{array}$ & + & $\begin{array}{c}\text { Создание акционерной } \\
\text { стоимости }\end{array}$ & $\begin{array}{l}\text { Не отвергается } \\
\quad(\mathrm{p}<0,05)\end{array}$ \\
\hline H5 & Государственная собственность & - & $\begin{array}{c}\text { Создание акционерной } \\
\text { стоимости }\end{array}$ & $\begin{array}{c}\text { Не отвергается } \\
(\mathrm{p}<0,1)\end{array}$ \\
\hline H6 & $\begin{array}{c}\text { Мажоритарный } \\
\text { иностранный акционер }\end{array}$ & + & $\begin{array}{c}\text { Создание акционерной } \\
\text { стоимости }\end{array}$ & $\begin{array}{c}\text { Не отвергается } \\
(\mathrm{p}<0,1)\end{array}$ \\
\hline $\mathrm{H} 7$ & $\begin{array}{c}\text { Концентрированность } \\
\text { структуры собственности }\end{array}$ & - & $\begin{array}{c}\text { Создание акционерной } \\
\text { стоимости }\end{array}$ & Отвергается \\
\hline H8 & Размер компании & + & $\begin{array}{c}\text { Создание акционерной } \\
\text { стоимости }\end{array}$ & $\begin{array}{l}\text { Не отвергается } \\
(\mathrm{p}<0,1)\end{array}$ \\
\hline H9 & Возраст компании & - & $\begin{array}{c}\text { Создание акционерной } \\
\text { стоимости }\end{array}$ & $\begin{array}{l}\text { Не отвергается } \\
\quad(\mathrm{p}<0,1)\end{array}$ \\
\hline
\end{tabular}

\section{Надежность полученных результатов}

Отметим результаты ряда диагностических тестов. Были рассчитаны VIF-факторы (Variance Inflation Factor - VIF) для тестирования гипотезы о наличии мультиколлинеарности. Значение VIF-факторов для независимых переменных во всех моделях ниже 1.5 (за исключением дамми-переменных времени и отраслей); среднее значение VIF во всех моделях ниже 3, что позволяет отклонить гипотезу о мультиколлинеарности.

Результаты теста Бройша-Пагана на гетероскедастичность на 1\%-ном уровне подтверждают наличие гетероскедастичности во всех моделях; для экономических данных редко следует ожидать гомоскедастичности остатков. Для устранения проблемы гетероскедастичности в линейных регрессиях использовались значения двустороннего t-теста для $\beta$ коэффициентов на основе робастных стандартных ошибок, скорректированных на гетероскедастичность по методу Уайта.

Что касается возможных проблем, связанных с эндогенностью, вызванных неучтенными факторами (omitted variables), то, во-первых, мы используем широкий набор контрольных переменных, во-вторых, модели с фиксированными эффектами позволяют учесть влияние 
инвариантных во времени индивидуальных эффектов компаний, важных с точки зрения создания акционерной стоимости.

Также следует отметить ряд ограничений проведенного анализа. Как уже отмечалось, из-за недоступности многих данных ряд переменных (структура собственности, риск) могут не совсем качественно аппроксимировать изучаемые характеристики. Во-вторых, даже формируя выборку из набора наиболее крупных компаний, мы не свободны от той проблемы, что акции ряда компании низколиквидны и данные по их рыночной стоимости могут существенно разниться с их фундаментальной стоимостью. В-третьих, мы отдаем себе отчет в том, что регрессии отражают корреляции, а не казуальность исследуемых зависимостей.

\section{Заключение}

В рамках проведенного эмпирического анализа детерминантов создания акционерной стоимости российскими компаниями в терминах стоимостных мультипликаторов цена/балансовая стоимость, цена/прибыль, акционерная доходность были получены следующие основные результаты:

- $\quad$ макроэкономическая конъюнктура выступает более мощным детерминантом создания акционерной стоимости по сравнению с индивидуальными характеристиками компании;

- $\quad$ такие финансовые драйверы стоимости, как доходность, политика в области структуры финансирования, инвестиционная активность и уровень рисковости позитивно коррелированы с созданием акционерной стоимости;

- характеристики структуры собственности выступают детерминантами стоимости компании; государственные компании торгуются с некоторым дисконтом, а представленные в структуре собственности крупным акционером-нерезидентом - с некоторой премией к рынку;

- уровень концентрации собственности не связан с относительной рыночной оценкой компании, однако негативно коррелирован с величиной акционерной доходности;

- $\quad$ размер, возраст компании, отраслевые эффекты статистически значимо связаны с индикаторами создания акционерной стоимости;

- $\quad$ индивидуальные характеристики компаний имеют большое значение в относительной оценке стоимости компании рынком и существенно меньшее значение в объяснении акционерной доходности.

\section{Список литературы}

1. Ивашковская И.В., Кукина Е.Б. Детерминанты экономической прибыли крупных российских компаний // Корпоративные финансы. 2009. №4. С. 18-33.

2. Сафиуллин М.Р., Анкудинов А.Б., Лебедев О.В. Исследование мотивов и факторов инвестиционной активности предприятий // Проблемы прогнозирования. 2013. № 4. С. 126-138.

3. Ankudinov, A.B., Safina, L.A. (2011), Investment in Innovations as Value Driver: Review of Empirical Studies and Analysis of Impact on Value Creation in Emerging Markets, International Journal of Humanities and Social Science, 5(1) (2011) 48-52.

4. Bai, Y., Green, C. (2011), Determinants of Cross-Sectional Stock Return Variations in Emerging Markets, Empirical Economics, 1(41) (2011) 81-102.

5. Bartov, E., Mohanram, P., Seethamraju, C. (2002), Valuation of Internet Stocks - an IPO Perspective, Journal of Accounting Research, 40 (2002) 321-346.

6. Cameron, A., Trivedi, P. (2009), Microeconometrics Using Stata. Stata Press.

7. Chen, L., Zhao, X. (2006), On the Relation Between the Market-to-Book Ratio, Growth Opportunity, and Leverage Ratio, Finance Research Letters, 3 (2006) 253-266.

8. Chen, P., Zhang, G. (2007), How Do Accounting Variables Explain Stock Price Movements? Theory and Evidence, Journal of Accounting and Economics, 43 (2007) 219-244. 
9. Chua, C.T., Eun, C.S., Lai, S. (2007), Corporate valuation around the world: the effects of governance, growth, and openness, Journal of Banking \& Finance, 31 (2007) 35-56.

10. Fama, E., French, K. (1996), Multifactor Explanations of Asset Pricing Anomalies. Journal of Finance, 1(51) (1996) 55-84.

11. Farinas, J.C., Moreno, L. (2000), Firms' Growth, Size and Age: A Nonparametric Approach, Review of Industrial Organization, 17 (2000) 249-265.

12. Gou, R., Lev, B., Zhou, N. (2005), The Valuation of Biotech IPOs, Journal of Accounting Auditing and Finance, 20 (2005) 423-459.

13. Hall, B. (1993), The Stock Market's Valuation of R\&D Investment during the 1980's, American Economic Review, 2(83) (1993) 259-264.

14. Hogan, C., Lewis, C.M. (2005), Long-Run Investment Decisions, Operating Performance and Shareholder Value Creation of Firms Adopting Compensation Plans Based on Economic Profits, Journal of Financial and Quantitative Analysis, 27 (2005) 261-284.

15. Huergo, E., Jaumandreu, J. (2004), Firms' Age, Process Innovation and Productivity Growth, International Journal of Industrial Organization, 22 (2004) 541-559.

16. Kleidon, A. (1986), Variance Bounds Tests and Stock Price Valuation Models, Journal of Political Economy, 5(94) (1986) 953-1001.

17. Koller, T., Goedhart, M., Wessels, D. (2010), Valuation: Measuring and Managing the Value of Companies. Wiley Finance, 5th Edition.

18. La Porta, R., Lopez de Silanes, F., Shleifer, A., Vishny, R. (2002), Investor Protection and Corporate Valuation, Journal of Finance, 57 (2002) 1147-1170.

19. Malighetti, P., Meoli, M., Paleari, S., Redondi, R. (2011), Value Determinants in the Aviation Industry, Transportation Research Part E: Logistics and Transportation Review, 3(47) (2011) 359-370.

20. Margaritis, D., Psillaki, M. (2010), Capital Structure, Equity Ownership and Firm Performance, Journal of Banking \& Finance, 3(34) (2010) 621-632.

21. Meoli, M., Paleari, S., Vismara, S. (2009), IPO Valuation of European Pyramidal Groups, Banking and Finance Review, 1 (2009) 17-34.

22. Olsen, E., Plaschke, F., Stelter, D. (2006), Spotlight on Growth: The Role of Growth in Achieving Superior Value Creation. Value Creators Report, The Boston Consulting Group.

23. Pandey, I. (2005), What Drives Shareholder Value, Asian Academy of Management Journal of Accounting and Finance, 1 (2005) 105-120.

24. Pedersen, T., Thomsen, S. (2003), Ownership Structure and Value of the Largest European Firms: the Importance of Owner Identity, Journal of Management and Governance, 7 (2003) 27-55.

25. Rappaport, A. (1998), Creating Shareholder Value: The New Standard for Business Performance. New York: The Free Press, 2nd edition.

26. Rapp, M., Schellong, D., Schmidt, M., Wolff, M. (2011), Considering the Shareholder Perspective: Value-Based Management Systems and Stock Market Performance, Review of Managerial Science, 1(5) (2011) 171-194.

27. StataCorp., (2009), Stata Longitudinal Data Reference Manual: Release 11.0. StataCorporation, College Station, Texas. 